\title{
TATA Box
}

National Cancer Institute

\section{Source}

National Cancer Institute. TATA Box. NCI Thesaurus. Code C13293.

A consensus sequence found in the promoter region of most genes transcribed by eukaryotic RNA polymerase II. It is found about 25 nucleotides before the site of initiation of transcription and has the consensus sequence: 5' TATAAAA 3'. This sequence seems to be important in determining accurately the position at which transcription is initiated. 